\title{
An Overview of Trust Models and Proposal of New Model Based on Reputation for Resource Selection in Grid Computing
}

\author{
Vivekananth.P
}

\begin{abstract}
Grid computing is distributed computing taken to the next evolutionary level. It facilitates the sharing of computer resources, allowing users to discover and use remote resources. The goal is to create the illusion of a simple yet large and powerful self managing virtual computer out of a large collection of connected heterogeneous systems sharing various combinations of resources. Users are able to submit jobs to remote resources and have no explicit control over the resources. A central problem for grid services is how to gain confidence that a remote system is performing in accordance with their norms. The effective and competent exploitation of grid computing services needs sophisticated and secured resource management systems. The wide range of selection and the high degree of strangeness leads to the problem in secured selection of resources grid. Without the assurance of a higher degree of confidence relationship, efficient resource allocation and utilization cannot be attained. In recent times, with larger applications in ecommerce and on-line communities, reputation mechanisms have become one of the most important techniques underpinning the distributed application and system safety. This paper investigates such reputation based trust models in the presence of malicious entities which give deliberately wrong feedbacks. We have proposed a new approach in this paper, which intends to offer trust and reputation provide a measure for resource selection in grid computing.
\end{abstract}

\section{INTRODUCTION}

Computational Grids enable the coordinated and aggregated use of geographically distributed resources, often owned by autonomous organizations, for solving large-scale problems in science, engineering, and commerce. However, application composition, resource management and scheduling in these environments is a complex undertaking. This is due to the geographic distribution of resources that are often owned by different organizations having different usage policies and cost models, and varying loads and availability patterns. To address these resource management challenges, we have developed a distributed computational economy framework for quality of servicedriven resource allocation and regulation of supply and demand for resources. In the open Grid environments, it is necessary to build the mechanism of evaluating reputation especially after the combination of Grid computing and economy.

Resources and security guarantee are the two fundamental requirements in Grid applications

Vivekananth.P, Lecturer-IT Department, St Joseph College of Engineering and Technology, Dar-Es-Salaam, Tanzania
Coordinated resource sharing and problem resolving in dynamic, multiinstitutional virtual organizations are the actual and specific problems which underlies the grid concept. Once infected shared grid resources through malicious codes planted by intruders possibly will spoil other applications running on the same Grid platform. The concerned sharing is not primarily file exchange but rather direct access to computers, software, data and other resources since it is required by a range of collaborative problem-solving and resource-brokering strategies emerging in industry, science and engineering. The wide range of selection and the high degree of strangeness leads to the problem in secured selection of the resources in grid. Without the assurance of a higher degree of trust relationship, competent resource allocation and utilization can not be attained. In recent times, with larger applications in ecommerce and on-line communities, reputation mechanisms have become one of the most important techniques underpinning the distributed application and system safety for its better scalability and flexibility. The rest of the chapters are organized as follows. Section 2 explains about the reputation systems, Section 3 discusses about literature survey, section 4 discusses about the model which uses reputation for resource selection and section 5 is the conclusion part.

\section{TRUST AND REPUTATION SYSTEMS}

Trust

Trust is a mechanism for reducing risk in unknown situations and expectation about behavior.

Reputation

Reputation refers to the value given to the entity (it may be a resource, service, user) based on the trust exhibited by it in the past. It reflects the perception that one has of another's intentions and norms.

The difference between trust and reputation can be illustrated by the following statements:

- "I trust you because of your good reputation."

- "I trust you despite your bad reputation."

The first sentence says that the first party believes the second one since the second one has a very good reputation. The good reputation may be obtained from one's own experience or from other's feed backs. The second sentence says that the first one believes the second in spite of the bad recommendations from others . This may be due to the strong belief or trust the first one has on the second. Personal experience typically carries more weight than second hand trust referrals or reputation, but in the absence 
of personal experience, trust often has to be based on referrals from others.

Reputation can be considered as a collective measure of trustworthiness (in the sense of reliability) based on the referrals or feed backs from members in the same community. An individual's subjective trust can be derived from a combination of received referrals and personal experience.

Reputation systems provide a way for building trust through social control by utilizing community based feedback about past experiences of peers to help making recommendation and judgment on quality and reliability of the transactions.

\section{REVIEW OF LITERATURE}

Li xiong and liu present [5] a reputation-based framework They claim that feed back values only are not enough for the calculation of trust and reputation. Y. Wang and J. Vassileva [6] propose a reputation model based on Bayesian network. According to their model the peers needs are different in different situations. Selcuk et al. suggests in [7] a reputation based trust management system in which the reliability is calculated based on previous transactions. Ayman Tajeddine et al. in [8] propose a very impressive reputation based trust model. In this approach the initiator host calculates reputation value of target host based on its previous experiences and gathered feedbacks from other hosts. F.Azzedin,M.Maheswaran [9] discuss about managing trust in grid by proposing a behavior trust management model. Trust levels are graded from a to $f$. Both direct and indirect trust are considered .

Gui Xiaolin, Xie Bing [10] propose a trust model based on behavior tracks. Attenuation function is corporated for decaying factor. Baolin Ma et al in [11] present a reputation based trusted model. Their model considers both direct feed back and feed back from other entities Direct trust is given with more weightage than the indirect score. Beulah kurian, Gregor von laszewki [12] provide a way for efficient resource selection. Their approach is similar to Azzedin approach [9] except for a new parameter "context".

\section{TRust Model fOr Grid RESOURCE SELECTION}

The proposed work is an enhancement of the existing model [8] that uses both direct trust and indirect trust. Direct trust is calculated from the transactions which are done directly by the initiator and is given higher weightage. Indirect trust is measured by getting feed backs from entities in the same domain and also from other domains. In the basic model the credibility of the recommenders feedback is estimated by considering different parameters such as similarity, activity and specificity.

The existing model took all the feedbacks into consideration while calculating the trust values. In the proposed model we assume that there can be a few malicious entities that can give wrong feedbacks about other entities. Even if single entity is giving a wrong feedback, it is sufficient to alter the decision from one state (grant) to another (not grant). In the real world we expect a set of malicious entities trying to disrupt the smooth functioning of the grid system by false reporting by giving false feedbacks. They can do so because the feedbacks that such entity gives are based on an entity's own evaluation .

The case for the modification of the existing method can be put forth as follows: Suppose A is the initiator and he wants to get feedbacks about a potential entity P. B and C have already transacted business with $\mathrm{P}$. A would like to use the feedbacks of $\mathrm{B}$ and $\mathrm{C}$ about $\mathrm{P}$, so as to determine whether to shortlist $\mathrm{P}$ as a candidate provider or drop him from listing.

The existing method simply uses the scores given by B and $\mathrm{C}$ and evaluates the trustworthiness of the provider as a function of above feedbacks. We go on to ask the questions - are the feedbacks given by entities are reliable? Unbiased? Trustworthy?

We can answer the above question if $\mathrm{A}, \mathrm{B}$, and $\mathrm{C}$ have given feedbacks about some common entities say E1, E2, E3, E4 and E5. A compares his feedbacks about these common entities with those given by $\mathrm{B}$ and $\mathrm{C}$. If there is a positive correlation then $\mathrm{A}$ takes the feedback back into account; and if the correlation is $<=0$ A ignores the corresponding feedbacks. For example if A's evaluations regarding entities E1, E2, E3, E4 and E5 are 4.8, 4, 3.6, 2.4 $\& 2$ and the evaluations of $\mathrm{B}$ and $\mathrm{C}$ respectively are 4.2, 3.9, 3.5, 2.5, 2.1 and 2.9, 2.7, 3, 3.5, 4.2 then A will not consider the feedback of $\mathrm{C}$.

Thus A the initiator entity can evaluate the trustworthiness of provider I, based on views of colleagues, whose evaluation schemes are similar to his. The correlation can be obtained by any of the standard methods available such as Pearson Product Moment Correlation, Spearman rank Order Correlation (rho) or the Kendall rank order Correlation (tau) and we have chosen Spearman's Rank Coefficient. Thus even if an entity tries to play havoc by giving false or unreliable feedback values they can be identified and eliminated from consideration.

In this paper, I focus only on eliminating unreliable feedback values from adversely affecting trust calculations. Quarantining such false feedback providers, if such actions are found to the deliberate is an issue that is to be considered separately and it is beyond the scope of this paper.

Ranking:

Since the feedbacks are collected from different domains, there is a chance of getting biased inputs. The feedbacks are sorted and rank is assigned. Rank correlation is calculated. If the result is positive then that entities feedback will be taken. Otherwise feedback values will not be considered. Only the feedbacks of entities with positive correlation are considered for calculating reputation.

Similarity $=1-6 \Sigma \mathrm{d}_{\mathrm{i}} 2 / \mathrm{n}(\mathrm{n} 2-1)$

number interactions by recommenders activity $=$

Total number interactions by all recommenders Number of interactions with initiator

Specificity $=$ 
hosts

Total number of interactions with all other

Credibility $=\mathrm{a} *$ similarity $+\mathrm{b} *$ activity $+\mathrm{c} *$ specificity where $\mathrm{a}>\mathrm{b}>\mathrm{c}$ and $\mathrm{a}+\mathrm{b}+\mathrm{c}=1$

A. Computation of reputation:

Consider the scenario where entity $\mathrm{x}$ wants to interact with entity $\mathrm{y}$ to complete some task. $\mathrm{X}$ wants to measure the trustworthiness of $\mathrm{y}$. The direct trust is calculated based upon the behavior of target entity on direct transactions. Then it inquires reputation of $y$ from the entities in the same domain and from other domain. The reputation will be calculated from the formula given below.

Rep $\mathrm{y} / \mathrm{x}_{\mathrm{k}}=\mathrm{u} *$ direct trust $+\mathrm{v} *$ indirect $1+\mathrm{w} *$ indirect 2 Where $\mathrm{u}+\mathrm{v}+\mathrm{w}=1$ and $\mathrm{u}>\mathrm{v}>\mathrm{w}$.

\section{$\sum \alpha_{\mathrm{i}}$ rep y/xi}

$\mathrm{i} \neq \mathrm{k}$

indirect $1=$

$\Sigma \alpha_{i}$

$\mathrm{i} \neq \mathrm{k}$

$\Sigma \beta_{\mathrm{j}}$ rep y/xi

$\mathrm{j} \neq \mathrm{k}$

indirect $2=$

\section{$\Sigma \beta_{\mathrm{j}}$}

$\mathrm{j} \neq \mathrm{k}$

$\alpha, \beta$ are credibility factors .

\section{CONCLUSION}

Grid resource selection is becoming more and more important topic, a number of problems still remain to be tackled by the current Grid solutions. Group-oriented security and distributed system behavior conformance are identified among the essential requirements for Grid resource selection. The proposed model removes the biased feed backs thereby improves the selection procedure.

\section{REFERENCES}

[1] A.Arenas "State of art survey on trust and security in grid computing system " march 2006

[2] Gheorghe Cosmin Silaghi, Alvaro E. Arenas, Luis Moura Silva," Reputation-based trust management systems and their applicability to grids "CoreGRID Technical Report Number TR-0064 February 23 , 2007

[3] Marcim Adamski, Alvaro Arenas, Angelos Bilas “ "Trust and Security in Grids: A State of the Art" CoreGRID White Paper Number WHP-0001 May 26, 2008

[4] A. Abdul-Rahman and S. Hailes. "Supporting trust in virtual communities". In HICSS '00: Proceedings of the 33rd Hawaii International Conference on System Sciences-Volume 6, page 6007, Washington, DC, USA, 2000. IEEE Computer Society.

[5] L. Xiong, and L. Liu, "PeerTrust: Supporting Reputation-Based Trust for Peer-to-Peer Electronic Communities," IEEE Transactions on Knowledge and Data Engineering, Vol. 16, No. 7, July 2004.

[6] Y. Wang and J. Vassileva, "Trust and Reputation Model in Peer-toPeer Networks," Proceedings of the Third International Conference on Peer-to-Peer Computing (P2P'03), 2003.

[7] A. Selcuk, E. Uzun, and M. Pariente, "A Reputation-Based Trust Management System for P2P Networks," IEEE International Symposium on Cluster Computing and the Grid 2004.

[8] Ayman Tajeddine Ayman Kayssi Ali Chehab Hassan Artail "A Comprehensive Reputation-Based Trust Model for Distributed Systems “ IEEE 2005.

[9] F.Azzedin,M.Maheswaran "Evolving and managing trust in grid computing system" IEEE CCECE,2002.

[10] Gui Xiaolin, Xie Bing "Study on behavior based trust model in grid security system "Proceedings of the 2004 IEEE International Conference on Services Computing (SCC'04).

[11] Baolin Ma, Jizhou Sun, Ce Yu " Reputation-based Trust Model in Grid Security System “, Aug. 2006, Volume 3, No.8 (Serial No.21) Journal of Communication and Computer, ISSN1548-7709, USA.

[12] Beulah kurian, Gregor von laszewki "Reputation based grid resource selection 